\title{
Lumbalgia en docentes universitarios por teletrabajo durante la pandemia por SARSCOV-2
}

\author{
Lic. Omar Páez Villaseñor \\ quiropavo@gmail.com \\ Universidad Estatal del Valle de Toluca \\ Estado de México, México \\ Mtra. Sandra Alicia Peñaloza Pérez \\ ellis_fenix@hotmail.com \\ Universidad Estatal del Valle de Toluca \\ Mtro. Efrén González Herrera \\ odiseo2010@live.com.mx \\ Universidad Estatal del Valle de Toluca
}

\section{RESUMEN}

El teletrabajo ha dejado una serie de estragos tanto físicos y psicológicos en los docentes universitarios desde que comenzó la pandemia, debido a que muchos de ellos pasan muchas horas sentados frente a o un ordenador y por la misma razón ha reducido su tiempo de actividad física, aunado al estrés que podría estar sufriendo debido al aumento de la carga laboral. El objetivo de esta investigación es identificar si las malas posturas que adoptan los docentes universitarios durante el teletrabajo están relacionadas con la lumbalgia o simplemente esta relacionado al tiempo que pasan sentados frente a un ordenador, métodos se realizó un estudio de descriptivo cohorte transversal, el universo se constituyó por docentes de la Universidad Estatal del Valle de Toluca en un total de 133 docentes. La muestra se conformó por 70 docentes, 25 docentes de la licenciatura en Quiropráctica, 25 de la carrera de Acupuntura Humana Rehabilitatoria y 20 de la carrera de Gerontología. Los resultados de la investigación se obtuvieron mediante la aplicación de una encuesta online, mismo que se tabularon en programa estadístico SSPS versión 20 y se presentaron en tablas estadísticas con su respectivo análisis, se concluye que si existe una relación entre las malas posturas, con la sintomatología de la lumbalgia inespecífica que presentan los docentes.

Palabras Clave: lumbalgia, teletrabajo, malas posturas 


\title{
Low back pain in university professors due to teleworking during the SARSCOV-2 pandemic
}

\begin{abstract}
Teleworking has left a series of physical and psychological factors on university teachers since the pandemic began, because many of them spend many hours sitting in front of or at a computer and for the same reason have reduced their physical activity time, coupled with to the stress you might be experiencing due to the increased workload. The objective of this research is to identify if the bad postures that university teachers adopt during teleworking are related to low back pain or simply related to the time they spend sitting in front of a computer, methods a descriptive cross-sectional cohort study was carried out, the universe It was constituted by teachers from the State University of the Valley of Toluca in a total of 133 teachers. The sample was made up of 70 teachers, 25 teachers from the Chiropractic career, 25 from the Human Rehabilitation Acupuncture carrer and 20 from the Gerontology carrer. The results of the investigation were obtained through the application of an online survey, which were tabulated in the statistical program SSPS version 20 and presented in statistical tables with their respective analysis, it is concluded that if there is a relationship between bad postures, with the symptoms of nonspecific low back pain presented by teachers.
\end{abstract}

Keywords: low back pain, teleworking, bad postures

Artículo recibido: 02 enero 2022 Aceptado para publicación: 28 enero 2022 Correspondencia: quiropavo@gmail.com Conflictos de Interés: Ninguna que declarar 


\section{INTRODUCCIÓN}

El teletrabajo se ha implementado con mayor frecuencia, a causa de la pandemia por SARS COV-2, tras un largo confinamiento se ha iniciado una revolución industrial, la pandemia ha demostrado que el 70\% de los trabajos, pueden realizarse de forma remota, sin embargo, solo 2 de cada 10 empresas cuentan con la cultura laboral y digital necesaria para implementarlo de manera eficiente.

EL modo de trabajo cambio y creo nuevas rutinas para estar a salvo en casa, ocasionando mayor índice de sedentarismo tras permanecer largas horas sentado frente a computadoras, pantallas; aumentado la probabilidad de lumbalgia por malas posturas en el home office.

El dolor de la espalada baja, o, lumbalgia es uno de los padecimientos con mayor frecuencia; alrededor del $80 \%$ de la población sufrirá dolores de espalda en algún momento de su vida, lo que supone un gran problema para el trabajador sus familiares y la sociedad ya que esta implica gastos inmensurables al sector salud.

Se trata de un problema muy frecuente que afecta aproximadamente a 80 de cada 100 personas. La lumbalgia se define principalmente como un síndrome doloroso localizado en la región lumbar con irradiación eventual a la región glútea, las caderas o la parte distal del abdomen generalmente se presenta después de realizar un gran esfuerzo con una postura inadecuada, como por ejemplo, después de cargar cosas muy pesadas sin protección de la columna o después de una caída o golpe muy fuerte. (IMSS I. M., 2014) La mayor parte de lumbalgias se originan por fuerzas ejercidas directamente sobre la columna, mediante la acción de diversos movimientos, cargas excesivas o posturas peligrosas en el trabajo, causando bajas laborales, así como baja productividad y pérdidas económicas para las empresas.

En la presente investigación se incluye el resultado de las encuestas realizadas a personas que ha realizado el teletrabajo o home office y así informar a la población que labora de esta manera, como es que la Quiropráctica pretende ser una opción en la prevención y tratamiento de la lumbalgia.

DESARROLLO

\section{Material y Métodos}


La presente investigación fue aplicada a los docentes de Universidad Estatal del Valle de Toluca, de los cuales se registraron un total 70 docentes, 25 docentes de la licenciatura en Quiropráctica, 25 de la carrera de Acupuntura Humana Rehabilitatoria y 20 de la carrera de Gerontología, se aplica 1 encuesta validada con 21 reactivos, se recolectó la información para describir las condiciones en las que los docentes realizan el teletrabajo en sus casas, y así poder establecer una relación entre las malas posturas que adoptan y la incidencia de lumbalgia. En seguida se muestra la información arrojada por la encuesta.

Tabla 1

\begin{tabular}{l|l|l}
\multicolumn{3}{c}{ Genero } \\
& $\mathrm{N}$ & $\%$ \\
\hline Masculino & 32 & $45.7 \%$ \\
\hline Femenino & 38 & $54.3 \%$ \\
\hline
\end{tabular}

Nota: En la población tomada como estudio se señala que de 70 docentes de nivel superior el $45.7 \%$ es género masculino mientras que $54.3 \%$ es género femenino En esta tabla se evidencia como está distribuida la muestra por género done el porcentaje de docentes del sexo femenino es superior a los del género masculino 


\section{Tabla 2}

\section{Edacd}

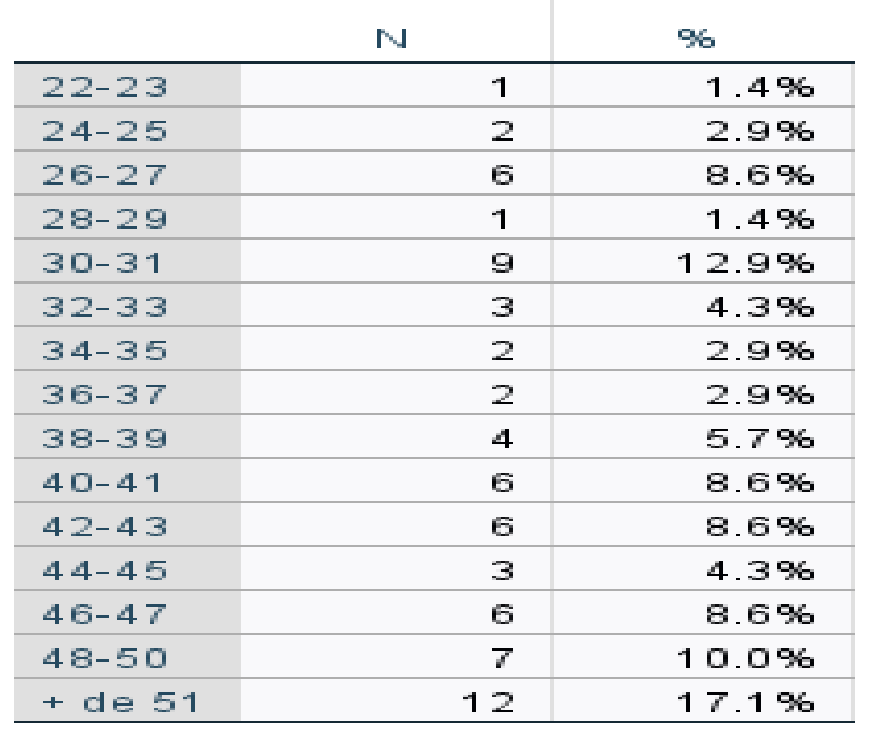

Nota: En la población tomada como estudio se señala que de 70 docentes de nivel superior el 17.1\% tienen mas de 51 años, $12.9 \%$ de 30 a 31 años y el $10.0 \%$ de 48 a 50 años

\section{Tabla 3}

\section{¿Cuanto tiempo llevas laborando como docente?}

\begin{tabular}{|l|r|r|} 
& $N$ & \multicolumn{1}{c}{$\%$} \\
\hline 0-6 meses & 2 & $2.9 \%$ \\
\hline 12- 24 meses & 10 & $14.3 \%$ \\
\hline 2-4 años & 11 & $15.7 \%$ \\
\hline 4-6años & 12 & $17.1 \%$ \\
\hline + de 6 años & 35 & $50.0 \%$ \\
\hline
\end{tabular}

\section{¿Cuanto tiempo llevas realizando home office 0 teletrabajo?}

\begin{tabular}{l|r|l} 
& \multicolumn{1}{c}{$N$} & \multicolumn{1}{l}{$\%$} \\
\hline 4-6 meses & 7 & $10.0 \%$ \\
\hline 7-12 meses & 13 & $18.6 \%$ \\
\hline + de 1 año & 50 & $71.4 \%$ \\
\hline
\end{tabular}

Nota: En la población tomada como estudio se señala que de 70 docentes de nivel superior el 50.0\% lleva laborando como docente más de 6 años y el 71.4\% lleva más de 1 años realizando home ofrece por la pandemia por SARS COV -2 


\section{Tabla 4}

¿Cuentas con un horario

fijo para lealizar el

teletrabajo?

\begin{tabular}{ll|l} 
& \multicolumn{1}{l}{$N$} & \multicolumn{1}{l}{$\%$} \\
\hline Si & 39 & $55.7 \%$ \\
\hline No & 31 & $44.3 \%$ \\
\hline
\end{tabular}

\section{¿Cuantas horas laborales, permaneces en una sola posición?}

\begin{tabular}{|l|r|r|} 
& $N$ & \multicolumn{1}{c}{$\%$} \\
\hline 30 min- 1 hora & 6 & $8.6 \%$ \\
\hline $2-4$ horas & 39 & $55.7 \%$ \\
\hline $5-7$ horas & 15 & $21.4 \%$ \\
\hline $8-10$ horas & 8 & $11.4 \%$ \\
\hline+12 horas & 2 & $2.9 \%$ \\
\hline
\end{tabular}

Nota: En la población tomada como estudio se señala que de 70 docentes de nivel superior el $55.7 \%$ cuenta con un horario fijo de trabajo y el $55.7 \%$ permanece en una misma posición entre 2 y 4 horas seguidas.

Tabla 5

\section{Seleccione el lugar donde realiza sus actividades}

\begin{tabular}{l|r|l} 
& N & \multicolumn{1}{l}{$\%$} \\
\hline Dormitorio & 8 & $11.4 \%$ \\
\hline Sala & 7 & $10.0 \%$ \\
\hline Comedor & 12 & $17.1 \%$ \\
\hline Estudio & 31 & $44.3 \%$ \\
\hline Mesa y silla improvisada & 12 & $17.1 \%$ \\
\hline
\end{tabular}

Nota: En la población tomada como estudio se señala que de 70 docentes de nivel superior el $44.3 \%$ realiza sus actividades en un estudio. 


\section{Tabla 6}

Que tan probable es que realice sus actividades en los siguientes lugares DORMITORIO (CAMA)

\begin{tabular}{lr|l} 
& N & \multicolumn{1}{l}{$\%$} \\
\hline Muy probable & 8 & $11.4 \%$ \\
\hline Poco probable & 33 & $47.1 \%$ \\
\hline Nada probable & 29 & $41.4 \%$ \\
\hline
\end{tabular}

\section{Que tan probable es que realice sus actividades en los siguientes lugares COMEDOR}

\begin{tabular}{l|l|l} 
& N & $\%$ \\
\hline Muy probable & 32 & $45.7 \%$ \\
\hline Poco probable & 26 & $37.1 \%$ \\
\hline Nada probable & 12 & $17.1 \%$ \\
\hline
\end{tabular}

\begin{tabular}{|l|l|l|} 
& N & \multicolumn{1}{l}{$\%$} \\
\hline Muy probable & 15 & $21.4 \%$ \\
\hline Poco probable & 37 & $52.9 \%$ \\
\hline Nada probable & 18 & $25.7 \%$ \\
\hline
\end{tabular}

Nota: En la población tomada como estudio se señala que de 70 docentes de nivel superior el $47.1 \%$ es poco probable que realice sus labores en la cama, el $45.7 \%$ es muy probable que realice sus labores en el comedor, y $52.9 \%$ es poco probable que realice sus actividades en la sala (sofá) 
¿Dispone del espacio

necesario para la

estación de trabajo?

\begin{tabular}{ll|l}
\multicolumn{2}{c}{ N } & \multicolumn{1}{l}{$\%$} \\
\hline Si & 43 & $61.4 \%$ \\
\hline No & 27 & $38.6 \%$ \\
\hline
\end{tabular}

¿El teclado y el ratón están

al mismo nivel, a la altura

de los codos de la persona,

con los codos flexionados en

90 ${ }^{\circ}$

\begin{tabular}{ll|l}
\multicolumn{2}{c|}{$\mathrm{N}$} & $\%$ \\
\hline Si & 28 & $40.0 \%$ \\
\hline No & 42 & $60.0 \%$
\end{tabular}

\section{¿Dispone de espaciolibre \\ debajo del escritorio que \\ permita movilizar las \\ piernas y detrás del \\ escritorio para la \\ movilidad de la silla?}

\begin{tabular}{ll|l}
\multicolumn{2}{c|}{ N } & \multicolumn{1}{l}{$\%$} \\
\hline Si & 50 & $71.4 \%$ \\
\hline No & 20 & $28.6 \%$ \\
\hline
\end{tabular}

\section{¿La silla cuenta con características y dimensiones adecuadas \\ de acuerdo a las \\ características \\ antropomtricas de la \\ persona?}

\begin{tabular}{l|l|l}
\multicolumn{2}{c|}{ N } & \multicolumn{1}{l}{$\%$} \\
\hline Si & 21 & $30.0 \%$ \\
\hline No & 49 & $70.0 \%$ \\
\hline
\end{tabular}

Nota: En la población tomada como estudio se señala que de 70 docentes $\overline{\text { de nivel }}$ superior el $61.4 \%$ dispone de espacio para la estación de trabajo, el 70.0\% cuenta con una silla con características y dimensiones adecuadas, ,el 71.4\% dispone de espacio libre debajo del escritorio para tener movilidad, y el 60.0\% no tiene ellos codos a la altura del ratón y el teclado .

\section{Tabla 8}

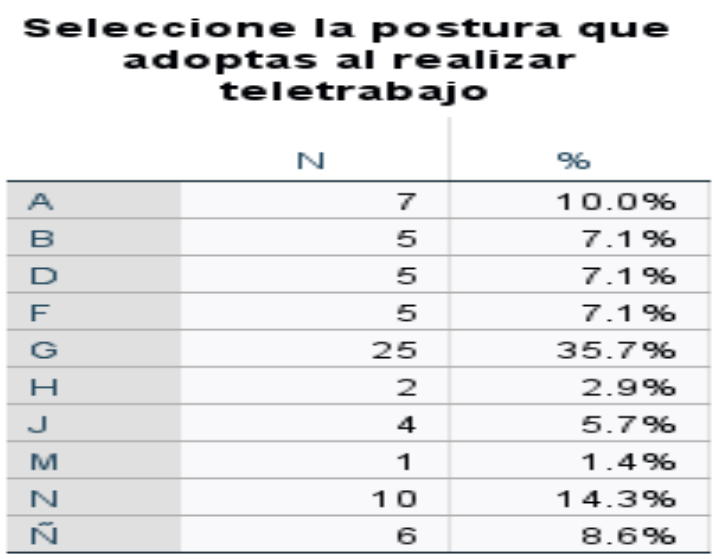

Nota: En la población tomada como estudio se señala que de 70 docentes de nivel superior el $35.7 \%$ adopta la postura indicada en la figura 16 


\section{Ilustración 1}
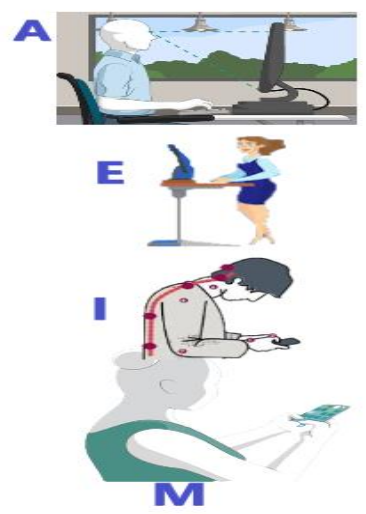
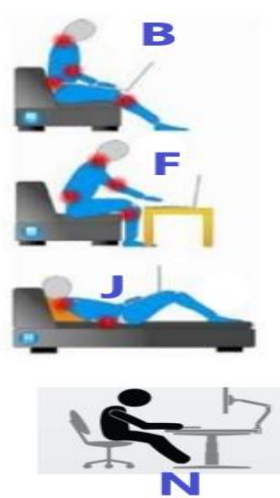
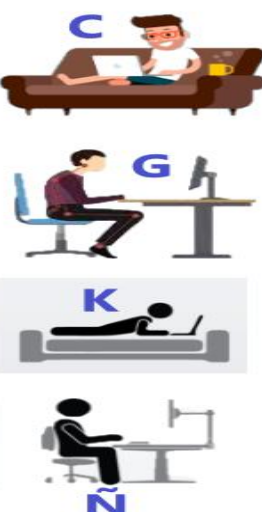
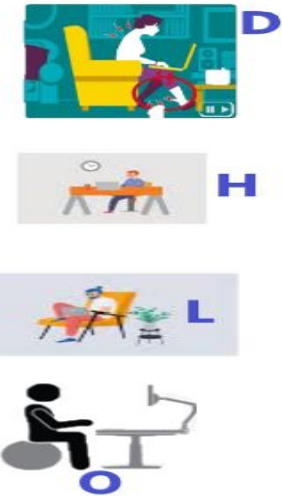

Notas: las posturas correctas están representadas con las letras $A, E, H, \tilde{N} y O$, mientras que las letras $B, C, D, F, G, I, J, K, L, M, N$, representan las malas posturas que se adoptan al realizar teletrabajo.

Tabla 9

\section{¿Realizas alguna}

actividad física?

\begin{tabular}{ll|l}
\multicolumn{2}{c}{ N } & \multicolumn{1}{l}{$\%$} \\
\hline Si & 41 & $58.6 \%$ \\
\hline No & 29 & $41.4 \%$ \\
\hline
\end{tabular}

Nota: En la población tomada como estudio se señala que de 70 docentes de nivel superior el $58.6 \%$ realiza actividad física, mientras que el $41.4 \%$ no.

\section{Tabla 10}

\section{¿Como es su dolor?}

\begin{tabular}{lr|r}
\multicolumn{1}{c}{$N$} & \multicolumn{1}{l}{$\%$} \\
\hline Quemante & 7 & $10.0 \%$ \\
\hline Punzante & 19 & $27.1 \%$ \\
\hline Eléctrico & 8 & $11.4 \%$ \\
\hline Sordo & 8 & $11.4 \%$ \\
\hline Lancinante & 2 & $2.9 \%$ \\
\hline Palpitante & 4 & $5.7 \%$ \\
\hline Perdidos Sistema & 22 & $31.4 \%$ \\
\hline
\end{tabular}

Nota: En la población tomada como estudio se señala que de 70 docentes de nivel superior el $27.1 \%$ padece un tipo de dolor punzante y un $31.4 \%$ no ha padecido dolor lumbar. 
Tabla 11

\begin{tabular}{|c|c|c|c|}
\hline & & $N$ & 96 \\
\hline 1 & & 9 & 12.996 \\
\hline 2 & & 1 & 1.496 \\
\hline 3 & & 3 & 4.396 \\
\hline 4 & & 10 & $14.3 \%$ \\
\hline 5 & & 5 & 7.196 \\
\hline 6 & & 8 & 11.496 \\
\hline 7 & & 10 & 14.396 \\
\hline 8 & & 4 & 5.796 \\
\hline 9 & & 2 & 2.996 \\
\hline 10 & & 2 & 2.996 \\
\hline Perdidos & Sistema & 16 & $22.9 \%$ \\
\hline
\end{tabular}

Nota: En la población tomada como estudio se señala que de 70 docentes de nivel superior la predominancia en el nivel de dolor se encuentra en $14.3 \%$ en un dolor $4 / 10$, en $14.3 \%$ un dolor $7 / 10,12.9 \%$ un dolor $1 / 10,11.4 \%$ en un dolor de $6 / 10$ mientras que el $22.9 \%$ no presenta dolor lumbar. 
Tabla 12

\section{¿Previo a la pandemia padeciste \\ lumbalgia?}

\begin{tabular}{|c|c|c|c|}
\hline & & & $\%$ \\
\hline $\mathrm{Si}$ & & 34 & $48.6 \%$ \\
\hline No & & 35 & $50.0 \%$ \\
\hline Perdidos & Sistema & 1 & $1.4 \%$ \\
\hline
\end{tabular}

Nota: En la población tomada como estudio se señala que de 70 docentes de nivel superior el $48.6 \%$ padeció dolor lumbar previo a la pandemia por SARS COV-2

\section{Tabla 13}

\section{Durante la cuarentena ha padecido lumbalgia}

\begin{tabular}{|c|c|c|c|}
\hline & & & $\%$ \\
\hline $\mathrm{Si}$ & & 42 & $60.0 \%$ \\
\hline No & & 9 & $12.9 \%$ \\
\hline Perdidos & Sistema & 19 & $27.1 \%$ \\
\hline
\end{tabular}

Nota: En la población tomada como estudio se señala que de 70 docentes de nivel superior el $60.0 \%$ padeció dolor lumbar durante la pandemia por SARS COV-2

\section{Tabla 14}

\section{¿Cuantas veces a padecido lumbalgia desde la cuarentena?}

\begin{tabular}{|l|r|r|} 
& $N$ & \multicolumn{1}{l}{$\%$} \\
\hline 1 vez por semana & 13 & $18.6 \%$ \\
\hline 2-3veces por semana & 16 & $22.9 \%$ \\
\hline $4-5$ veces por semana & 4 & $5.7 \%$ \\
\hline Toda la semana & 1 & $1.4 \%$ \\
\hline $1-2$ veces por mes & 10 & $14.3 \%$ \\
\hline Perdidos Sistema & 26 & $37.1 \%$ \\
\hline
\end{tabular}

Nota: En la población tomada como estudio se señala que de 70 docentes de nivel superior el 22.9\% padeció dolor lumbar de 2 a 3 veces por semana durante la pandemia por SARS COV-2 


\section{Tabla 15}

\section{En las siguientes actividades, selecione la frase que más se parezca a su situación Al sentarse}

\begin{tabular}{|l|l|l|} 
& \multicolumn{1}{l}{ N } & \multicolumn{1}{l|}{$\%$} \\
\hline $\begin{array}{l}\text { Puedo estar en cuaqier tipo de silla el } \\
\text { tiempo que quiera }\end{array}$ & 13 & $18.6 \%$ \\
\hline $\begin{array}{l}\text { Puedo estar sentado en mi silla } \\
\text { favorita el tiempo que quiera }\end{array}$ & 5 & $7.1 \%$ \\
\hline $\begin{array}{l}\text { Puedo estar sentado pero me genera } \\
\text { a aumenta dolor }\end{array}$ & 27 & $38.6 \%$ \\
\hline El dolor no me permite estar sentado & & \\
\hline Perdidos Sistema & 3 & $4.3 \%$ \\
\hline
\end{tabular}

Nota: En la población tomada como estudio se señala que de 70 docentes de nivel superior el $38.6 \%$ puede estar sentado pero le genera dolor, mientras que el $31.4 \%$ no presenta dolor lumbar

\section{Tabla 16}

\section{En las siguientes actividades, selecione la frase que más se parezca a su situación Al dormir}

\begin{tabular}{|l|c|c|}
\hline & N & $\%$ \\
\hline El dolor no me impide dormir bien & 39 & $55.7 \%$ \\
\hline Puedo dormir solo tomando pastillas & 7 & $10.0 \%$ \\
\hline El dolor me impide totalmente dormir & 8 & $11.4 \%$ \\
\hline Perdidos Sistema & & \\
\hline
\end{tabular}

Nota: En la población tomada como estudio se señala que de 70 docentes de nivel superior el $55.7 \%$ el dolor no le impide dormir, mientras que el $22.9 \%$ no presenta dolor lumbar 


\section{Tabla 17}

\section{En las siguientes actividades, selecione la frase que más se parezca a su situación Al estar de pie yo}

\begin{tabular}{l|r|l|} 
& N & \multicolumn{1}{l}{$\%$} \\
\hline $\begin{array}{l}\text { Puedo estar de pie tanto tiempo sin } \\
\text { que me aumente el dolor }\end{array}$ & 23 & $32.9 \%$ \\
\hline $\begin{array}{l}\text { Puedo estar de pie tanto tiempo como } \\
\text { quiera, pero aumenta el dolor }\end{array}$ & 17 & $24.3 \%$ \\
\hline El dolor no me permite estar de pie & 6 & $8.6 \%$ \\
\hline Perdidos Sistema & 24 & $34.3 \%$ \\
\hline
\end{tabular}

Nota: En la población tomada como estudio se señala que de 70 docentes de nivel superior el $32.9 \%$ puede estar de pie tanto tiempo sin que le aumente el dolor mientras que el $34.3 \%$ no presenta dolor lumbar

\section{Tabla 18}

\section{¿Realizaste o realizas algún tratamiento?}

\begin{tabular}{lr|r} 
& $N$ & \multicolumn{1}{l}{$\%$} \\
\hline Medico & 9 & $12.9 \%$ \\
\hline Rehabilitación & 4 & $5.7 \%$ \\
\hline Quiropráctico & 19 & $27.1 \%$ \\
\hline Ejercicios y estiramientos & 14 & $20.0 \%$ \\
\hline Perdidos Sistema & 24 & $34.3 \%$ \\
\hline
\end{tabular}

Nota: En la población tomada como estudio se señala que de 70 docentes de nivel superior el $34.3 \%$ no realiza ningún tratamiento, el $27.1 \%$ tiene tratamiento quiropráctico y el $20.0 \%$ realiza ejercicios y estiramientos.

\section{Tabla 19}

\begin{tabular}{l|ccl}
\multicolumn{1}{c|}{ Encuesta } & Si & \multicolumn{2}{c}{ No } \\
\hline Pregunta 22 & $34 / \mathrm{E}=43.7$ & $35 / \mathrm{E}=25.3$ & 69 \\
Pregunta 23 & $42 / \mathrm{E}=32.3$ & $9 / \mathrm{E}=18.7$ & 51 \\
Total & 76 & 44 & 120
\end{tabular}

Elaborado por: Madai Ferreyra Balandrán y Estefanía González de la Cruz 2021 Notas: Pregunta 22 ¿Previo a la pandemia padeciste lumbalgia?, pregunta 23 ¿durante la cuarentena ha padecido lumbalgia? 
Tabla 10

\begin{tabular}{l|llll} 
Encuesta & \multicolumn{1}{l}{ Si } & No & Total \\
\hline Pregunta 15 & $43 / \mathrm{E}=29$ & $27 / \mathrm{E}=41$ & 70 \\
Pregunta 20 & $15 / \mathrm{E}=29$ & $55 / \mathrm{E}=41$ & 70 \\
Total & 58 & 82 & 170
\end{tabular}

Elaborado por: Madai Ferreyra Balandrán y Estefanía González de la Cruz 2021

Notas: Pegunta 15 ¿dispone de un espacio para la estación de trabajo? „Pregunta 20Selecciona la postura que adoptas al realizar teletrabajo o home office

\section{CONCLUSIONES}

Después de valorar la población de estudio se puede señalar que existe relación entre las malas posturas, con la sintomatología de la lumbalgia Inespecífica, ya que el $38.5 \%$ realizan sus labores en un estudio o escritorio y $61.4 \%$ lo realizan en diferentes áreas del hogar, el $50.7 \%$ de estos cuentan área adecuada que cumple las características necesarias para desempeñar sus actividades sin embargo solo el $21.5 \%$ de los encuestados selecciono posturas adecuadas mientras que el $78.5 \%$ seleccionaron posturas inadecuadas. En el proceso de valoración a través de la encuesta se identificó que el grado de dolor que presentan los docentes con $14.3 \%$ un EVA7/10, con $14.3 \%$ un EVA 4/10, con $12.9 \%$ un EVA $1 / 10$ un $22.9 \%$ no presenta dolor lumbar; asimismo se evaluó el tipo de dolor que más presentan es punzante con un $27.1 \%$.

De los docentes evaluados el $54.3 \%$ padeció dolor lumbar previo y/o durante la cuarentena, el $31.4 \%$ no padeció lumbar en la cuarentena y el $14.25 \%$ no presento ningún dolor en la espalda.

De estos un $27.1 \%$ tomaron un tratamiento quiropráctico, un $20.0 \%$ ejercicios y estiramientos, mientras que $34.3 \%$ no presento ningún dolor en la espalda.

Para verificar la hipótesis planteada, se trabaja con la prueba de chi cuadrado Qué es un estilógrafo no paramétrico de distribución libre, que permite establecer correspondencia entre valores observados y esperados, llegando a la comprobación de distribuciones enteras.

\section{Ecuación 1}

$$
X^{2}=£(\underline{(O-E) 2})
$$


$\mathrm{X}^{2}=$ Chi cuadrado

$£=$ sumatoria

$\mathrm{O}=$ datos observados

$\mathrm{E}=$ datos esperados

\section{Pregunta 22}

¿Previo a la pandemia padeciste lumbalgia?

\section{Pregunta 23}

¿Durante la cuarentena ha padecido lumbalgia?

\section{Revisar tabla 26}

$\mathrm{X}^{2}=13.7$

$\mathrm{P}=<.005$

estándar $=3.84$

\section{Pegunta 15}

¿dispone de un espacio para la estación de trabajo?

\section{Pregunta 20}

Selecciona la postura que adoptas al realizar teletrabajo o home office

\section{Revisar tabla 27}

$X^{2}=23.06$

$\mathrm{P}=<.005$

Con ambas verificaciones las diferencias entre las observaciones son drásticamente significativas.

El valor de $\mathrm{x}^{2} \mathrm{t}=3.84, \mathrm{x} 2=13.7$ y 23.06 , por tanto a la regla de decisión establecida se rechaza la hipótesis nula y se acepta la hipótesis alterna, es decir se confirma que la sintomatología de la lumbalgia inespecífica está relacionada con las malas posturas de los docentes de nivel superior en el teletrabajo o home office durante la pandemia por SARS Cov-2 


\title{
APÉNDICES Y ANEXOS
}

\section{Anexo 1 : Encuesta}

\section{"Lumbalgia inespecifica debido a malas posturas en el teletrabajo en docentes de nivel superior"}

\author{
Enimato 5 isth
}

gretar par w whth.

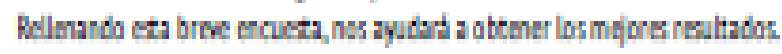

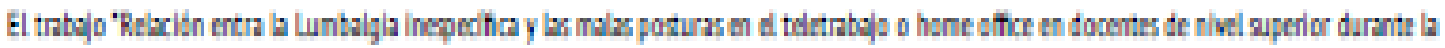

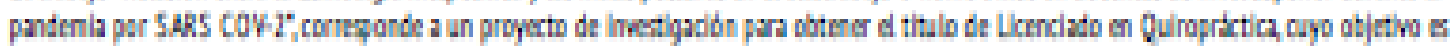

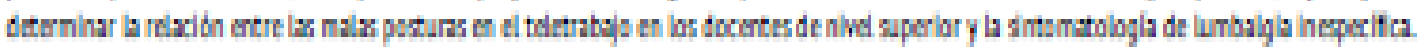

La participacớn en este estudio es estrictamente voluntaria, La infomaciọ́n recolectada será confidencial, con fines estadisțticos y de investigación. Si esta dispuesto a participar seleccione donde comesponda.

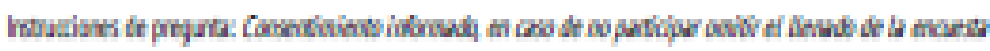

$\bigcirc \mathrm{O}$

Genero

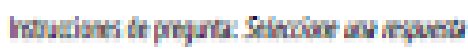

O fumaln: O Houln

\section{Edad}

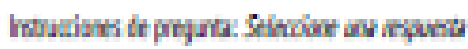
O 131901511
Onis
O 245
(3) 16
O 319
(j) 551
(a) 1245 a 55
(3) 5
() 1999
(3) 40-41
(1) 45
(3) 444
(1640 O450 O+dis 


\section{Zona de residencia}

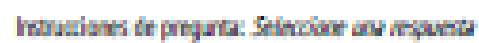

O thal $\mathrm{O}$ แั่aาa

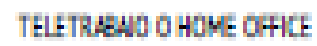

¿Cuánto tiempo llewas laborando como docente?

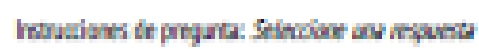

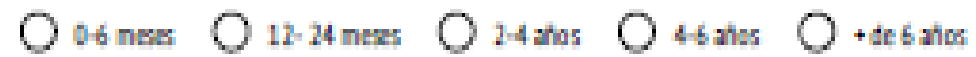

¿Cuánto tiempo llevas realizando home office o teletrabajo?

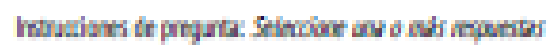

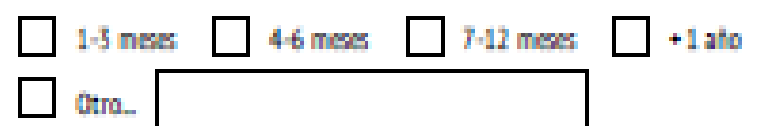

¿Cuenta con un horario fijo, para realizar el teletrabajo?

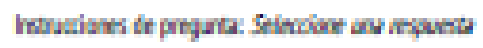

0 i $\mathrm{m}$

A partir de la Pandemia por SARS CoV-2, ¿Cuántas horas al día trabaja en la computadora y/0 celular?

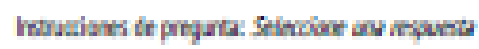

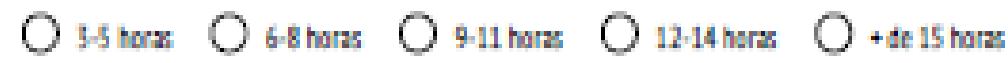

¿Cuántas horas laborales, pemaneces en una sola posición?

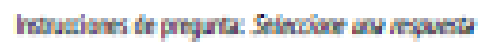

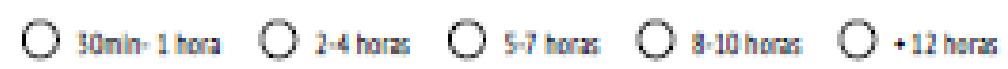


Seleccione el lugar donde realiza sus actividades

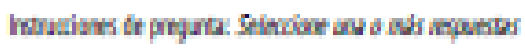

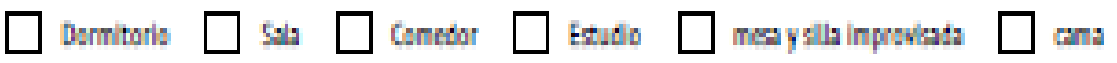

Que tan probable es que realice sus actividades en los siguientes lugares

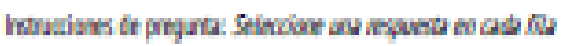

\begin{tabular}{|c|c|c|c|}
\hline & muip protable & poop probatis & rastapritable \\
\hline Dounterlo [ama) & $\square$ & $\square$ & $\square$ \\
\hline Conntor & $\square$ & $\square$ & $\square$ \\
\hline 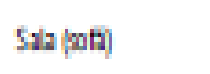 & $\square$ & $\square$ & $\square$ \\
\hline Etuduts & $\square$ & $\square$ & $\square$ \\
\hline
\end{tabular}

Dispone del espacio necesario para la estación dẹ trabajo?

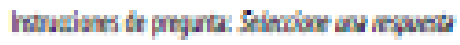

$\mathrm{O}_{\mathrm{O} i \mathrm{in}} \mathrm{O}$

LLa silla cuenta con caracteristicas y dimensiones adecuadas de acuerdo a las caracteristicas antropométricas de la persona (como asiento ajustable en altura, respaldo, decansabrazos ajustables en altura y base giratoria de cinco puntos de apoyo con ruedas, bordes redondeados, material transpirable?

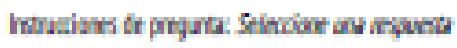

$O$ si $O$ k

Otro. 
¿Dispone de espacio libre debajo del escritorio que permita movilizar las piernas y detrás del escritorio para la movilidad de la silla?

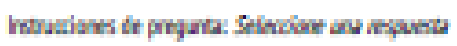

$\mathrm{O}_{\text {si }} \mathrm{O}_{\mathrm{No}}$

¿El teclado y el mouse están al mismo nivel, a la altura de los codos de la persona, con los codos flexionados en $90^{\circ}$ ?

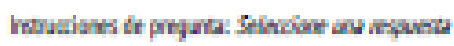

$\mathrm{O}$ si $\mathrm{ONo}$

¿El monitor está ubicado frente a la persona trabajadora, y el borde superior está al nivel de los ojos?

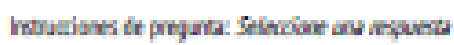

Osi $\mathrm{O}$ No

Selecciona las posturas que adoptas al realizar teletrabajo

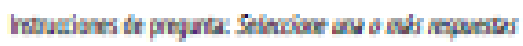

$\square$

$\square$ A $\square$ s $\square$ c $\square$ o $\square$ ह $\square$ \& $\square$ s

$\square \wedge \square$ o

¿Realizas alguna actividad fisica?

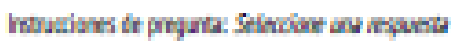

O $51 \quad$ ONo

Otro.

Esta seccion salo deberd ser contestata si padeces o padeciate lunbalgia (dolar en espalda ba/a) en casa de no haberla padecido onitir las slgalentes preguntas. 


\section{Comos sudolor}

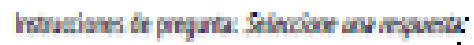

$\square$
O puenate
Purnite
Elintrio
() serd:
O Landate 0 palphite

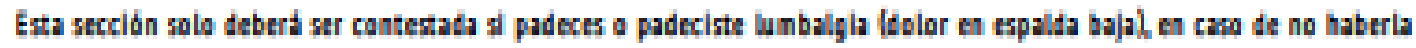
padecido amitir las sigulertes preguntas.

En las siquientes actividades, seleccionę la frase que más se parezca a su situación:

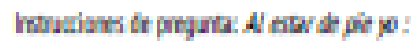

$\square$ Pude athr de ple tanto tiempo gh que ne aunatie did tolar

$\square$ Puade star de ple tanto timpo como qulari,

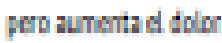

$\square$ Eldiber me menter sitir teple

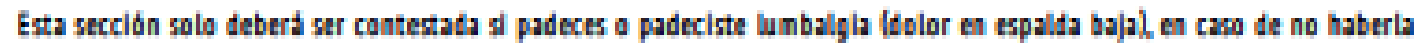
padecido amitir las sigulertes prequntas.

En las siguiente actividades, selecciones la frase que más se parezca a su situación:

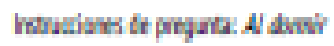

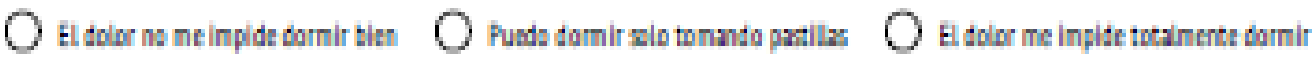

En las siguientes actividades, seleccione la frase que más se parezca a su situaciọn:

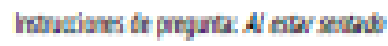

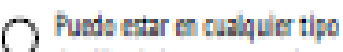 tesila ditampo cue quard
O Puade enar mathe en nille

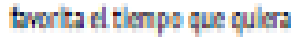 me gamen suments tular
Puedo etor wombin par: El tolar mo me pernitr etar mants

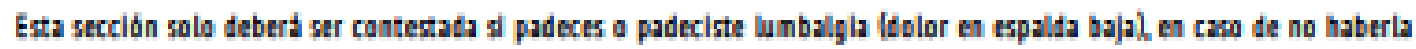
padecido amitir las sigulertes preguntas.
¿Realizaste o realizas algún tratamiento?

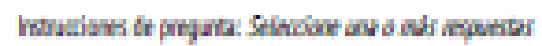
Mation
Ruhatitritn
Duropititio:
Eicridory otinniertor 


\section{Previo a la pandemia padeciste lumbalgia (dolor en espalda baja)}

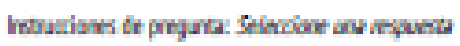

si $\bigcirc \mathrm{ns}$

Esta sección solo deberd ser contestata sil padeces o padeciste lumbalgia (dolor en espalda bajal en caro de no haberia padecido anitir las siguientes preguntas.

\section{Durante la cuarentena ha padecido lumbalgia(dolor en espalda baja)}

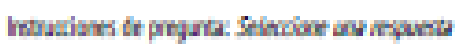

si $\mathrm{ONs}$

Esta sección solo deberd ser contestata sil padeces o padeciste lumbalgia (dolor en espalda bajal en caro de no haberla padecido amitir las siguientes preguntas.

Cuantas veces a padecido lumbalgia, durante la cuarentena.

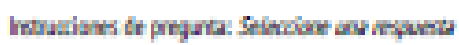

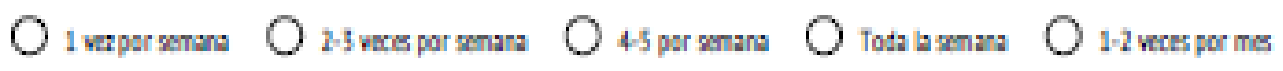

Esta sección solo debert ser contestata sil padeces o padeciste lumbalgia (dolor en espalta bajal en caso de no haberia padecido anitir las siguiertes preguntas.

\section{En una escala del 0 al 10 como considera su dolor}

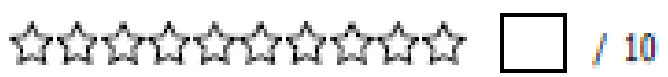

Esta sección solo debert ser contestata sil padeces o padeciste lumbalgia (dolor en espalta baja) en caso de no haberia padecido amitir las siguientes preguntas.

\section{ANEXO2}

\section{Bioética.}

Se incluyó el consentimiento informado para la participación encada encuesta aplicada, respetando el uso de datos personales 


\section{REFERENCIAS BIBLIOGRAFICAS}

¿Que es la medicina complementaria? (30 de 08 de 2017). Medicina complementaria y holistica. (Breastcancer.org, Productor) Obtenido de ¿Que es la medicina complementaria?:

http://www.breastcancer.org/es/tratamiento/medicina_comp/que-es

Balagué, F., Mannion, A., \& Pellise, F. (04 de 02 de 2012). Non- especific low back pain. Lancet, 482-491. doi:https://doi.org/10.1016/50140-6736(11)60610-7

Calvet, V., \& Marques, O. (2010). Guía de practica clinica para la lumbalgia insepecífica. Europan Commission Cost, Obtenido de http://www.webdelaespalda.org/cientifica/guias/guiascost.asp

Chou, R., Qaseem, A., Snow, V., Casey, D., Cross, J., \& Shekelle, P. (2007). Diagnosis and treatment of low back pin: a joint clinical practice. Guideline, American Collage of Physicians on American Pain Society, 147, 478-91.

Clara, E. (01 de 2001). Ergonomia y planificación. Elsevier, 20(1), 100-103. Obtenido de https://www.elsevier.es/es-revista-ofform-4-articulo-salud-laboral-ergonomiaplanificacion-13759

David, C. (2004). Principios y objetivos del tratamiento quiropráctico. En C. David, Quiropráctica (págs. 61-68). .: Temas de Navarra. Recuperado el 03 de 2021, de http://www.quiropráctica.com/wp-content/uploads/2015/09/quirobook0.8pdf

ENMT, E. d. (2015). Guía Practica de Salud Laboral para la valoración de Aptitud en Trabajadores con Riesgo de Exposición a carga Física. Asociación Española de Especialidades de Medicina del Trabajo , -.

IMSS, I. M. (19 de 03 de 2014). Salud en linea. Obtenido de Lumbalgia: www.imss.gob.mx/salud-en-linea/lumbalgia

IMSS, I. M. (02 de 10 de 2018). Unidad de comunicación. Obtenido de En el gobierno mas de 300 mil consultas por lumbalgia en 2017: https://wwwimss.gob.mx/prensa/archivo/201810/246

J Tenias, C. M. (2006). Absentismo Laboral por dolor de espalda en personal hospitalario. MApfre, S/N.

Laura, P., Robert, R., \& Andred, P. (04 de 2020). Ergonomía cvisual en el marco del teletrabajo quedate en casa. (INAHE, Ed.) Instituto de ambiente hábitat y energía, $1-9$. 
Mar't, V. G. (s.f.).

Martínez, V. G. (21 de Junio de 2016). Ausentismo Laboral y Salud: estudio de importancia en el teletrabajo. Retos, $\mathrm{S} / \mathrm{N}$.

Mary, L., \& Gavin, M. (05 de 2013). Seguridad y primeros auxilios. Recuperado el 03 de 2021, de medicina complementaria y alternativa: http://www.rchsd.org/healtharticles/medicina-complementaria-y-alternaiva/

Ministerio de salud. (. de . de s.f). Tipos de telerabajo. Obtenido de Ministerio de salud: https://www.ministeriodesalud.go.cr/index.php/teletrabajo/tipos-teletrabajo

Ministerio de trabajo y seguridad. (s.f). guía de salud ocupacional y prevenciíon de los riesgos en el teletrabajo. MTSS, 1-17. Obtenido de https://www.cso.go.cr/documentos-relevantes/manuales-

guias/guias/guiaadesaludocupaciuonalyprevencionsocialdelosriesgoseneltrabajo. pdf

O. A. (2009). Signos de alarma en la lumbalgia . $S / N$, s/n.

Osakidetza. (2007). Guía de Práctica Clínica sobre la lumbalgia. -: GNP.

Perez, J. (2018). Incidencia de lumbalgia y factores asociados a pacientes adultos que acuden al centro de salud del valle durante el mes de julio a febrero 2018. (F. d. medicas, Ed.) Universidad de cuenca, -. Obtenido de https://dspace.ucuenca.edu.ec/bitstream/123.456789/30230/1/PROYECTO\%20I NVESTIG4CIO\%C3\%93N.pdf

Poitras, S., Rossignol, M., Dionne , C., \& Tousignant, M. (2008). Interdisciplinary clinical model for the management of low back :in primary care. $B M C$, Musculoskelet discor, 9-54.

Secretaria del Trabajo y Prevencion Social. (12 de 01 de 2020). Secretaria del trabajo. Obtenido de entra en vigor reforma que regula el teletrabajo en mexico: https://www.gob.mx/stps/prensa/entra-en-vigor-reforma-que-regula-elteletrabajo-en-mexico

Uned. (. de . de s.f). Programa del teletrabajo. Obtenido de caracteristicas: http://www.uned.ac.cr/viplan/teletrabajo/que-esteletrabajo/tipos-de-teletrabajo

Vicente, M., Torres, J., Torres, A., Ramirez , M., \& Capdevila, I. (Julio-Diciembre de 2018). El teletrabajo en Salud Labloral aspectod médico-legales y laborales. CES Derechi, 9(2), 287,297. 
World federation of chiropractic. (. de . de 2009). World Federation of Chiropractic. Recuperado el 03 de 2021, de Datos sobre la quiropráctica: https://www.wfc.org/website/index.php?option=com_content\&view=article\&id $=122 \&$ itemid $=138 \&$ langises 